\title{
ONE-VARIABLE EQUATIONAL COMPACTNESS IN PARTIALLY DISTRIBUTIVE SEMILATTICES WITH PSEUDOCOMPLEMENTATION
}

\author{
SYDNEY BULMAN-FLEMING ${ }^{1}$ AND ISIDORE FLEISCHER ${ }^{1}$
}

\begin{abstract}
A universal algebra $A$ is called one-variable equationally compact if every system of equations with constants in $A$ involving a single variable $x$, every finite subsystem of which has a solution in $A$, has itself a solution in $A$. The one-variable equationally compact semilattices with pseudocomplementation $\langle S ; \wedge, *, 0\rangle$ which satisfy the partial distributive law $x \wedge(y \wedge z)^{*}=\left(x \wedge y^{*}\right) \vee$ $\left(x \wedge z^{*}\right)$ are characterized, and as a consequence we are able to describe the one-variable compact Stone semilattices. Similar considerations yield a characterization of the one-variable equationally compact Stone algebras, extending a well known result for distributive lattices.
\end{abstract}

The one-variable equationally compact semilattices with pseudocomplementation $\langle S ; \wedge, *, 0\rangle$ which satisfy the partial distributive law $x \wedge(y \wedge z)^{*}=(x \wedge$ $\left.y^{*}\right) \vee\left(x \wedge z^{*}\right)$ are characterized, and as a consequence we are able to describe the one-variable equationally compact Stone semilattices. Similar considerations yield a characterization of the one-variable equationally compact Stone algebras, extending a well known result for distributive lattices.

A universal algebra $A$ is called one-variable equationally compact if every system of equations with constants in $A$ involving a single variable $x$, every finite subsystem of which has a solution in $A$, has itself a solution in $A$. Note that the equations to be considered are equalities between one-variable polynomials with constants in $A$, i.e. expressions built up from the elements of $A$ and a single variable $\boldsymbol{x}$ by repeated application of the fundamental operations of $\boldsymbol{A}$. For more information on this notion and the more familiar one of equational compactness, consult [20], [22], or the appendix to [10] by G. H. Wenzel.

General references throughout this article are Grätzer [10] for Universal Algebra, and Birkhoff [5], Grätzer [11], or Balbes and Dwinger [2] for Lattice Theory. We shall consistently use the term semilattice to mean meet-semilattice unless the contrary is specifically indicated. A semilattice with pseudocomplementation is an algebra $\langle S ; \wedge, *, 0\rangle$ of type $\langle 2,1,0\rangle$ such that $\langle S ; \wedge, 0\rangle$ is a semilattice with least element 0 and $x \wedge s=0$ in $S$ iff $x<s^{*}$. The notation PCS will henceforth be used to denote the class of all such algebras and also as an abbreviation for

Received by the editors September 11, 1979.

AMS (MOS) subject classifications (1970). Primary 06A20; Secondary 06A25, 08A25.

Key words and phrases. Semilattice, pseudocomplement, equationally compact.

${ }^{1}$ This research was supported by grants from the National Research Council of Canada. The authors also benefited from the hospitality of the Department of Pure Mathematics, University of Waterloo, as extended by Professor Angus Kerr-Lawson, during preparation of a preliminary version of this paper. 
"semilattice with pseudocomplementation". In addition to the usual semilattice laws, the following rules of computation are often used:

(1) $x<x^{* *}$,

(2) $(x \wedge y)^{* *}=x^{* *} \wedge y^{* *}$,

(3) $x * *=x *$,

(4) $0 * *=0$,

(5) $x<0^{*}$ (and so $0^{*}$ is a largest element 1 ),

(6) $x<y$ implies $y^{*}<x^{*}$,

(7) $\left(\bigvee x_{i}\right)^{*}=\wedge x_{i}^{*}$ whenever $\bigvee x_{i}$ exists,

(8) $\left(\bigwedge x_{i}\right)^{*}=\left(\bigvee x_{i}^{*}\right)^{* *}$ whenever $\wedge x_{i}$ and $\bigvee x_{i}^{*}$ exist and the infinite form of (2) holds.

REMARK 1. That the dual of (7) may fail to hold even in a complete PCS and for a down-directed family $\left(x_{i}\right)$ may be seen by considering the PCS consisting of the negative integers in their usual order, with $-\infty$ adjoined. This example shows that in fact even (8) can fail in the absence of the infinite form of (2). It turns out that the equality $\bigvee x_{i}^{*}=\left(\bigwedge x_{i}\right)^{*}$ holds for all down-directed families $\left(x_{i}\right)$ in a complete PCS $S$, iff $S$ satisfies conditions (ii) and (iii) of Theorem 3 below (the straightforward proof is omitted).

One defines the set $B(S)$ (of closed elements of $S$ ) to be $\left\{x \in S: x^{* *}=x\right\}$ (which is also equal to $\left\{x^{*}: x \in S\right\}$ ). Properties (1)-(4) above imply that $* *$ is a meet- and 0-preserving closure operator. It is well known [9], [11], that if $a \dot{\vee} b$ is defined to be $\left(a^{*} \wedge b^{*}\right)^{*}$ for $a, b \in B(S)$ then $\langle B(S) ; \wedge, \dot{V}, *, 0,1\rangle$ is a Boolean algebra; moreover, the PCS $\left\langle B(S) ; \Lambda,{ }^{*}, 0\right\rangle$ is a retract of $S$ via the homomorphism $s \mapsto s^{* *}$. Since ${ }^{* *}$ is a closure operator, existing infima of families of closed elements are closed. If the join $V a_{i}$ of a family of closed elements exists in $S$ it fails in general to belong to $B(S)$; of course, in this case the join in $B(S)$ does exist and equals $\left(\mathrm{V} a_{i}\right)^{* *}$.

DefinImon 2. A PCS $\langle S ; \wedge, *, 0\rangle$ is called partially distributive if $x \wedge\left(y^{*}\right.$ $\left.\dot{\vee} z^{*}\right)=\left(x \wedge y^{*}\right) \vee\left(x \wedge z^{*}\right)$ for all $x, y, z \in S$.

The above definition is to be understood as asserting the existence (in the partial order derived from $\wedge$ ) of the join $\left(x \wedge y^{*}\right) \vee\left(x \wedge z^{*}\right)$ for all $x, y, z \in S$. Thus the operator $y \mapsto x \wedge y$, which is always a semilattice endomorphism on $S$, now restricts to $B(S)$ as a lattice morphism into $S$. Taking $x$ to be 1 shows that in a partially distributive PCS, $B(S)$ is in fact a sublattice of $S$ (although of course $S$ itself need not be a lattice). Note also that the left-hand side of the equality in Definition 2 can be written as $x \wedge(y \wedge z)^{*}$, as has already been done in the introductory paragraph. A law similar to our partial distributivity has been considered in [16].

A semilattice $\langle S ; \wedge\rangle$ is called distributive (cf. [11] or [15]) if $x>a \wedge b$ implies the existence of $a_{1}>a$ and $b_{1}>b$ with $x=a_{1} \wedge b_{1}$ (thus, in the terminology of [18], if every $a \wedge b$ is distributive). We shall here call a semilattice conditionally distributive if whenever $y \vee z$ exists, then $(x \wedge y) \vee(x \wedge z)$ exists for every element $x$ and equals $x \wedge(y \vee z)$. (This characterizes semilattices of subsets in which pairwise joins, whenever they exist, are unions; see [21] and, as noted in [8], 
the much earlier paper [18].) By specializing [18, Corollary 6.4], noting that the distributivity of existing joins follows from Lemma $4.23^{*}$ of the same paper (see also [1, Theorem 4.1 (ii)]), we find that every distributive semilattice is conditionally distributive. It can be shown furthermore that every conditionally distributive PCS in which the "Stone Identity" $x^{*} \vee x^{* *}=1$ holds is partially distributive. (Hence $B(S)$ is a sublattice of $S$-this generalizes $2.6[15]$.) Thus the class of partially distributive PCS's includes all Stone semilattices [15]; in fact, Stone semilattices are precisely those PCS's which are both distributive and partially distributive.

We now give the theorem characterizing the one-variable equationally compact partially distributive semilattices with pseudocomplementation.

THEOREM 3. Every one-variable equationally compact $P C S\left\langle S ; \wedge,{ }^{*}, 0\right\rangle$ satisfies the following three conditions:

(i) $S$ is a complete lattice such that for every $a \in S$ and every up-directed family $\left(x_{i}\right)$ in $S$, the equality $a \wedge \vee x_{i}=\bigvee a \wedge x_{i}$ holds.

(ii) $\bigwedge x_{i}^{* *}=\left(\bigwedge x_{i}\right)^{* *}$ for every family $\left(x_{i}\right)$ in $S$.

(iii) $\bigvee x_{i}^{* *}=\left(\bigvee x_{i}\right)^{* *}$ for every up-directed family $\left(x_{i}\right)$ in $S$.

Conversely, every partially distributive PCS satisfying conditions (i)-(iii) is onevariable equationally compact.

The necessity of (i)-(iii) follows immediately from [7] (taking a single distinguished endomorphism ${ }^{* *}$ in the Theorem and in Note (2) of that paper) wherein appropriate finitely solvable systems of one-variable equations are considered to give each condition. In fact, appropriate systems of equations for (i) are already contained in [12]. Proof of the sufficiency of (i)-(iii) for partially distributive $S$ is postponed to the Appendix section of this paper in order that we may now explore some of the consequences of Theorem 3.

The following remark results in an alternative formulation of Theorem 3 (note that a still different formulation is possible according to Remark 1 above).

ReMARK 4. In any one-variable equationally compact PCS, $B(S)$ is a complete $\bigwedge$-subsemilattice of $S$ in which up-directed joins agree with joins taken in $S$. If also (as in the partially distributive case) $B(S)$ is a sublattice of $S$ then it is in fact a complete sublattice, for in this case condition (iii) of Theorem 3 holds for arbitrary families $\left(x_{i}\right)_{i \in I}$ in $S$; simply observe that $\bigvee x_{i}=\bigvee x_{F}$ where $F$ ranges over the finite subsets of $I$ and $x_{F}=\bigvee_{i \in F} x_{i}$, so $\left(\bigvee x_{i}\right)^{* *}=\left(\bigvee x_{F}\right)^{* *}=\bigvee x_{F}^{* *}=$ $\bigvee x_{i}^{* *}$. (The last equality holds because $B(S)$ is a sublattice of $S$.)

COROLlary 5. A Stone semilattice $\langle S ; \wedge, *, 0\rangle$ is one-variable equationally compact iff it satisfies conditions (i)-(iii) of Theorem 2.

A Stone algebra is an algebra $\left\langle S ; \wedge, \vee,{ }^{*}, 0,1\right\rangle$ such that $\langle S ; \wedge, \vee, 0,1\rangle$ is a bounded distributive lattice, $\langle S ; \wedge, *, 0\rangle$ is a PCS and the identity $x^{*} \vee x^{* *}=1$ holds. For Stone algebras as well, $B(S)$ is a sublattice of $S$. (See [2] or [11].) With a minimal amount of extra effort, a characterization of the one-variable equationally compact Stone algebras can be given. 
TheOREM 6. Let $\left\langle S ; \wedge, \vee,{ }^{*}, 0,1\right\rangle$ be a Stone algebra. Then $S$ is one-variable equationally compact iff

(i) $S$ is complete,

(ii) $S$ satisfies the distributive laws $a \wedge \bigvee x_{i}=\bigvee a \wedge x_{i}$ and $a \vee \wedge x_{i}=$ $\wedge a \vee x_{i}$ for all $a \in S$ and all families $\left(x_{i}\right)$ in $S$,

(iii) $\bigwedge x_{i}^{* *}=\left(\bigwedge x_{i}\right)^{* *}$ and $\bigvee x_{i}^{* *}=\left(\bigvee x_{i}\right)^{* *}$ for all families $\left(x_{i}\right)$ in $S$.

Proof. The necessity of (i)-(iii) follows from a combination of the one-variable equational compactness of the associated meet- and join-semilattices, Theorem 3, and Remark 4.

Proof of the sufficiency of conditions (i)-(iii) is deferred to the Appendix.

Corollary 7 (Kelly [17], Beazer [4]). A distributive lattice $D$ is one-variable equationally compact iff it is complete and infinitely distributive, i.e. iff it satisfies conditions (i) and (ii) of Theorem 6.

Proof. The necessity of the conditions follows from the one-variable equational compactness of the associated meet- and join-semilattices.

To see that the conditions are also sufficient, simply observe that $D$ is a lattice retract of a one-variable equationally compact Stone algebra $S$ (formed by adjoining a new smallest element to $D$ ) and so is itself one-variable equationally compact.

Appendix. We present here proofs that the conditions of Theorem 3 and Theorem 6 are sufficient for the one-variable compactness of partially distributive PCS's and Stone algebras, respectively. Our approach is modelled after [12]; namely, we show that the solution sets of one-variable equations with constants are, under the assumed conditions, closed under formation of arbitrary infima and up-directed suprema, and then use these facts to deduce solvability from finite solvability.

LEMMA A.1. Every one-variable PCS polynomial with constants in an arbitrary PCS S may be written in at least one of the forms:

(1) $a \wedge\left(b^{*} \dot{\vee} x^{*}\right) \wedge\left(c^{*} \dot{\vee} x^{* *}\right)$

(2) $a \wedge x$,

for suitable elements $a, b, c \in S$.

Proop. The constant polynomial $a$ can be written in form (1), the polynomial $x$ in form (2), and the totality of expressions which can be written in form (1) or in form (2) is closed under $\wedge$ (by virtue of the identity $\left.x \wedge(y \wedge x)^{*}=x \wedge y^{*}\right)$ and * (inasmuch as all Boolean one-variable polynomials with constants in $B(S)$ occur for $a=1$ in form (1)).

LemMA A.2. Partial distributivity permits writing the form (1) of Lemma A.1 both as

$$
a \wedge\left(b^{*} \vee x^{*}\right) \wedge\left(c^{*} \vee x^{* *}\right)
$$


and as

$$
\left(d \wedge x^{*}\right) \vee\left(e \wedge x^{* *}\right)
$$

for suitable elements $a, b, c, d, e$.

Proor. Use the fact that a Boolean polynomial may be written in both conjunctive and disjunctive form and apply partial distributivity (recalling that in this case $\checkmark$ and $\dot{V}$ coincide for closed elements).

Observe that the form $\left(d \wedge x^{*}\right) \vee\left(e \wedge x^{* *}\right)$ which appears above is not formally a PCS polynomial with constants. The existence of all such joins (when $x$ is replaced by elements of $S$ ) is a consequence of partial distributivity, as noted earlier.

LeMma A.3. In a partially distributive PCS $\left\langle S ; \wedge,{ }^{*}, 0\right\rangle$ every one-variable PCS equation with constants in $S$ is equivalent to a conjunction of inequalities

$$
p<q
$$

where $p$ is of the form $a \wedge x, a \wedge x^{*}$ or $a \wedge x^{* *}$ and $q$ is of the form $b, x, b^{*} \vee x^{*}$ or $b^{*} \vee x^{* *}$. The nontautologous forms are all included among $p<b$ and $a \wedge x^{* *}$ $<x$.

Proof. One conducts a case-by-case examination of all possibilities determined by Lemma A.2. For example, $a \wedge x<b^{*} \vee x^{*}$ iff $a \wedge x<b^{*} \wedge x$ iff $a \wedge x<$ $b^{*}$, which is one of the forms $p<b$.

LEMMA A.4. In a partially distributive PCS satisfying conditions (i)-(iii) of Theorem 3, the solution set of any one-variable PCS equation with constants is closed under arbitrary meets and up-directed joins.

Proof. We need only show that the solution sets of inequalities of the forms $a \wedge x<b, a \wedge x^{* *}<b, a \wedge x^{* *}<x$ and $a \wedge x^{*}<b$ are closed under arbitrary meets and up-directed joins. Since the operators $x \mapsto x^{* *}$ and $x \mapsto a \wedge x$ preserve both these operations, only the closure under meets of the solution set of $a \wedge x^{*}<$ $b$ need be checked. Assuming that $\left(x_{i}\right)_{i \in I}$ is a family of solutions of this inequality, and that $F$ ranges over the finite subsets of $I$, we have

$$
\begin{aligned}
a \wedge\left(\wedge x_{i}\right)^{*} & =a \wedge\left(\bigwedge_{F} \bigwedge_{i \in F} x_{i}\right)^{*} \\
& =a \wedge\left(\bigvee_{F}\left(\bigwedge_{i \in F} x_{i}\right)^{*}\right)^{* *} \quad \text { (by equality (8) at the beginning) } \\
& =a \wedge \bigvee_{F}\left(\bigwedge_{i \in F} x_{i}\right)^{*} \quad \text { (by (iii)) } \\
& =\bigvee_{F} a \wedge\left(\bigwedge_{i \in F} x_{i}\right)^{*} \quad(\text { by (i)) } \\
& =\bigvee_{F} \bigvee_{i \in F} a \wedge x_{i}^{*}
\end{aligned}
$$

(partial distributivity in the form occurring on page 505)

$<b$

which is the required inequality. 
Proof of Theorem 3 (CONCLUded). We show that conditions (i)-(iii) of Theorem 3 are sufficient for the partially distributive PCS $S$ to be one-variable equationally compact. Let $\Sigma$ be any finitely solvable set of one-variable PCS equations with constants in $S$, let $\mathcal{F}$ denote the set of all finite subsets of $\Sigma$, and for $F \in \mathscr{F}$ let $a_{F}$ be the smallest common solution of all the equations in $F$ ( $a_{F}$ exists by Lemma A.4). Then $a=\bigvee_{F \in \mathcal{F}} a_{F}$, as the union of an up-directed family in $S$ which eventually includes a solution for each $\sigma \in \Sigma$, is a common solution of all the equations in $\Sigma$, by the preceding lemma.

Proof of Theorem 6 (CONCluded). The proof of Theorem 6 is entirely similar to that of Theorem 3 given above, once the analogue of Lemma A.4 for Stone algebras has been established. The latter task is accomplished by first observing that one-variable Stone algebra polynomials with constants may be written both in the form

$$
a \vee(b \wedge x) \vee\left(c \wedge x^{*}\right) \vee\left(d \wedge x^{* *}\right)
$$

and in the dual of this form. Thus any one-variable Stone algebra equation with constants may be resolved into a conjunction of simple inequalities similar to those obtained in Lemma A.3. Relying upon our preceding work for partially distributive PCS's and upon the known results for equationally compact (= one-variable equationally compact) semilattices, we find that the theorem will be proven if we show that the solution sets of inequalities of the types $a<b \vee x^{*}, a<b \vee x^{* *}$ and $a \wedge x^{* *}<b \vee x$ are also closed under formation of infima and up-directed suprema. To check this is by now routine and is therefore omitted.

Final Remark. Simple examples show that the study of equational compactness either for more-than-one-variable systems or for algebras which are not partially distributive is not feasible using this method. Indeed, in any Boolean algebra of more than one element, the solution set of the equation $x \dot{V} y=1$ is not closed under infima. The same is true of the solution set of $c \wedge x^{*}=x^{*}$ in the 5-element PCS obtained from the 4-element Boolean algebra, by adjoining a dense nonclosed element $c$. This algebra is equationally compact, distributive, but not partially distributive. In fact a PCS is partially distributive if and only if the solution sets of all equations of the form $a \wedge x^{*}=b \wedge x^{*}$ are closed under formation of pairwise meets.

\section{REFERENCES}

1. R. Balbes, $A$ representation theory for prime and implicative semilattices, Trans. Amer. Math. Soc. 136 (1969), 261-267.

2. R. Balbes and Ph. Dwinger, Distributive lattices, Univ. of Missouri Press, Columbia, Mo., 1974.

3. R. Balbes and A. Horn, Stone lattices, Duke Math. J. 37 (1970), 537-545.

4. R. Beazer, A characterization of complete, bi-brouwerian lattices, Colloq. Math. 29 (1974), 55-59.

5. G. Birkhoff, Lattice theory, 3rd ed., Amer. Math. Soc. Colloq. Publ., Vol. 25, Amer. Math. Soc., Providence, R. I., 1967.

6. S. Bulman-Fleming, On equationally compact semilattices, Algebra Universalis 2 (1972), 146-151.

7. S. Bulman-Fleming, I. Fleischer and K. Keimel, The semilattices with distinguished endomorphisms which are equationally compact, Proc. Amer. Math. Soc. 73 (1979), 7-10.

8. I. Fleischer, Embedding a semilattice in a distributive lattice, Algebra Universalis 6 (1976), 85-86.

9. O. Frink, Pseudocomplements in semilattices, Duke Math. J. 29 (1962), 505-514.

10. G. Grätzer, Universal algebra, 2nd ed., Springer-Verlag, New York, 1979. 
11. __ General lattice theory, Academic Press, New York, 1978.

12. G. Grätzer and H. Lakser, Equationally compact semilattices, Colloq. Math. 20 (1969), $27-30$.

13. G. T. Jones, Pseudocomplemented semilattices, Ph. D. Dissertation, U. C. L. A., 1972.

14. T. Katrinák, Pseudocomplementäre Halboerbände, Mat. Casopis Sloven. Akad. Vied. 18 (1968), 121-143.

15. , Die Kennzeichnung der distributiven pseudokomplementären Halboerbände, J. Reine Angew. Math. 241 (1970), 160-179.

16. __ Primitive Klassen von modularen S-algebren, J. Reine Angew. Math. 251 (1973), 55-70.

17. D. A. Kelly, A note on equationally compact lattices, Algebra Universalis 2 (1972), 80-84.

18. H. M. MacNeille, Partially ordered sets, Trans. Amer. Math. Soc. 2 (1937), 416-460.

19. H. P. Sankappanavar, A study of congruence lattices of preadocomplemented semilattices, Ph. D. Thesis, University of Waterloo, 1974.

20. J. Mycielski, Some compactifications of general algebras, Colloq. Math. 13 (1964), 1-9.

21. B. M. Schein, On the definition of distributive semilattices, Algebra Univeralis 2 (1972), 1-2.

22. W. Taylor, Review of several papers on equational compactness, J. Symbolic Logic 4 (1975), 88-92.

Departient of Mathematics, Winfrid Laurier Universtry, Watemlo0, Ontaro, N2L 3C5, Canada

Cantre de Rbcherchies Mathímatiques, Univerestié de Monmíal, Monmíal, P. Q., H3C 3J7, Canada 